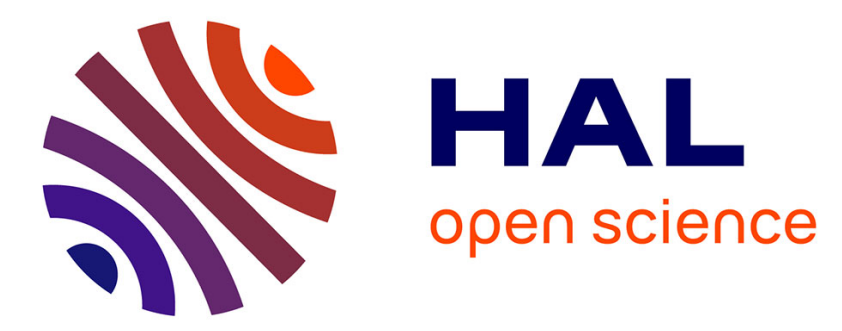

\title{
DÉTERMINATION DES VITESSES ULTRASONORES DANS LES TRĖS PETITS ÉCHANTILLONS PAR DIFFUSION BRILLOUIN
}

M. Fischer, Bernard Bonello, A. Polian, A. Zarembowitch

\section{- To cite this version:}

M. Fischer, Bernard Bonello, A. Polian, A. Zarembowitch. DÉTERMINATION DES VITESSES ULTRASONORES DANS LES TRĖS PETITS ÉCHANTILLONS PAR DIFFUSION BRILLOUIN. Journal de Physique Colloques, 1990, 51 (C2), pp.C2-45-C2-48. 10.1051/jphyscol:1990211 . jpa00230390

\section{HAL Id: jpa-00230390 https://hal.science/jpa-00230390}

Submitted on 1 Jan 1990

HAL is a multi-disciplinary open access archive for the deposit and dissemination of scientific research documents, whether they are published or not. The documents may come from teaching and research institutions in France or abroad, or from public or private research centers.
L'archive ouverte pluridisciplinaire HAL, est destinée au dépôt et à la diffusion de documents scientifiques de niveau recherche, publiés ou non, émanant des établissements d'enseignement et de recherche français ou étrangers, des laboratoires publics ou privés. 
COLLOQUE DE PHYSIQUE

Colloque C2, supplément au $n^{\circ} 2$, Tome 51, Février 1990

\title{
DETERMINATION DES VITESSES ULTRASONORES DANS LES TRÈS PETITS ECHANTILLONS PAR DIFFUSION BRILLOUIN
}

\author{
M. FISCHER, B. BONELLO, A. POLIAN* et A. ZAREMBOWITCH \\ Département de Recherches Physiques (CNRS URA 71), Université Pierre et \\ Marie Curle T. 22, 4, Place Jussieu, F-75252 París Cedex 05, France \\ * Laboratoire de physique des Milieux Condensés (CNRS URA 782), \\ Université Pierre et Marie Curie T. 13, 4, Place Jussieu, F-75252 Paris \\ Cedex 05, France
}

\begin{abstract}
Résumé - Nous décrivons un montage de diffusion Brillouin de grand contraste associé à une celluie à enclumes de diamant permettant d'etudier l'élasticité des matériaux soumis à de très fortes pressions hydrostatiques ( $P \geqslant 10 \mathrm{GPa}$ ). Nous présentons les résultats obtenus sur le titanate de strontium $\left(\mathrm{SrTiO}_{3}\right)$ entre la pression atmosphérique et $36 \mathrm{GPa}$. Ces résultats mettent en évidence à environ $6 \mathrm{GPa}$ un changement d'état que nous identifions comme êtant celui se produisant à pression atmosphérique et $105 \mathrm{~K}$.
\end{abstract}

Abstract - We describe a high contrast Brillouin spectrometer, associated with a dia mond anvils cell which allows us to study the elasticity of materials under high hydrostatic pressure. We present results obtained on $\mathrm{SrTiO}_{3}$ between atmospheric pressure and $36 \mathrm{GPa}$. These results show a phase transition at about $6 \mathrm{GPa}$ and room temperature. We identifie this instability as being that which occurs at atmospheric pressure and $105 \mathrm{~K}$.

\section{1 - INTRODUCTION}

La diffusion Brillouin est $1^{\prime}$ outil d'excellence lorsque $I^{\prime}$ on veut déterminer avec précision les vitesses d'ondes ultrasonores dans des échantillons transparents et de très petites dimensions (quelques dizaines de $\mu \mathrm{m}$ d'épaisseur). En particulier, les études sous haute pression $(P \geqslant 10 \mathrm{GPa})$ sont menées dans les cellules à enclumes de diamant /1/ sur des quantités de matière ne dépassant pas quelques microgrammes. La technique de diffusion Brillouin qui utilise une lumière focalisée, est alors parfaitement adaptée au faible volume expérimental dont on dispose dans ces cellules.

Les mesures acoustiques dans un échantillon soumis à une pression d'un ordre de grandeur comparable à celui de ses constantes élastiques revêt un grand intérêt, tant sur le plan théorique que sur le plan des applications. Elles permettent en particulier 1'étude des instabilités structurales qui peuvent apparaftre dans de telles conditions de contrainte.

Dans la première partie de cet article, nous décrivons un móntage de spectométrie Brillouin de très grand contraste, associé à une cellule à enclumes de diamant pour la génération des très hautes pressions. Ce dispositif nous a permis d'étudier l'élasticité du titanate de strontium $\left(\mathrm{SrTiO}_{3}\right)$ entre la pression atmospherique et $36 \mathrm{GPa}$, à la tempêrature ambiante. Les résultats expérimentaux qui sont décrits dans la seconde partie, mettent en évidence à environ $6 \mathrm{GPa} 1^{\prime}$ apparition d'un changement d'état accompagné d'une déformation 
structurale. Cette instabilité est identifiée comme étant celle se produisant à pression atmosphérique et $105 \mathrm{~K}$.

\section{2 - DISPOSITIF EXPERIMENTAL}

Le principe de fonctionnement d'une cellule à enclumes de diamant est illustré sur la figure 1. Un joint métallique est placé entre les faces planes (culasses) de deux diamants de taille brillant modifiée. L'échantillon monocristallin est placé dans un trou percé exactement au centre du joint avec un fluide transmetteur de la pression (le mélange 4:1 méthanol:éthanol dans notre cas) et un morceau de muby pour la mesure de la pression. Celle-ci est mesurée par le déplacement du pic de luminescence du ruby qui a été calibré jusqu'à $100 \mathrm{GPa} / 2 /$.

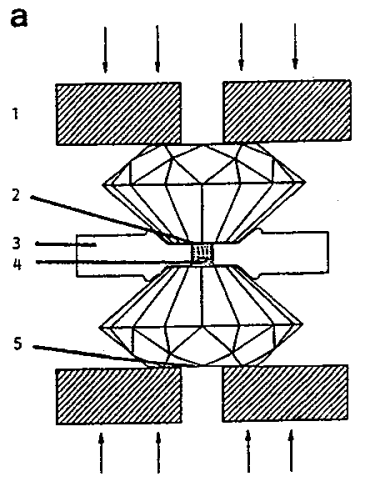

b

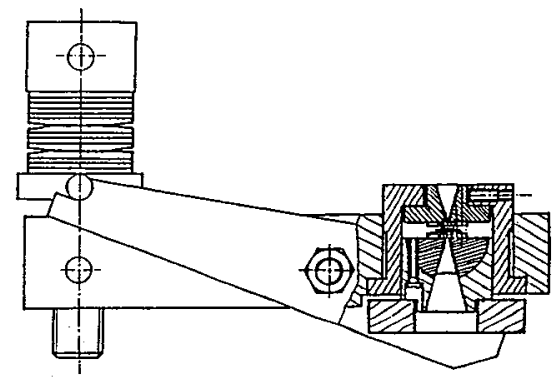

Fig. 1 - (a) Principe de fonctionnement d'une cellule à enclumes de diamant. 1, siège des diamants (carbure de tungstène); 2 , culasse $(0=0,6 \mathrm{~mm}) ; 3$, joint; 4, volume expérimental contenant I'échantillon, un ruby et le fluide transmetteur de pression; $5, \cdot \operatorname{table}(0=4 \mathrm{~mm})$. (b) cellule de type Block-Piermarini.

Le déplacement Brillouin étant maximum dans une direction de propagation faisant un anglé de $\pi$ radians avec la direction du faisceau lumineux incident (rétrodiffusion), c'est cette géométrie que nous avons adoptée. Le déplacement du nombre d'onde Brillouin $\Delta \sigma$ est alors relié à $l a$ vitesse $V$ de l'onde d'agitation thermique se propageant dans cette direction par:

$$
\Delta \sigma=\frac{2 \mathrm{n} \mathrm{V}}{\lambda_{0} \mathrm{c}}
$$

où $\mathrm{c}$ est la vitesse de la lumière dans le vide, n l'indice de réfraction du cristal pour la Iumière incidente de longueur d'onde dans le vide $\lambda_{0}$.

L'échantilion étudié étant petit et imparfait, la lumière diffusée élastiquement par la surface et les défauts peut être plus intense que les pics Brillouin. Dans de telles conditions, il faut améliorer le contraste de l'interféromètre en faisant passer plusieurs fois dans celui-ci la lumière diffusée par le cristal. On réalise ceci en utilisant des coins de cube qui réfléchissent le faisceau lumineux parallèlement à lui-même /3/. Notre dispositif est alors équivalent à cinq Faby-Pérot synchronisés en série. L'ensemble du montage est schématisé sur la figure 2, La lumière excitatrice est la raie à $514,5 \mathrm{~nm} \mathrm{~d}^{\dagger}$ un laser argon monomode, utilisé avec une puissance d'environ $100 \mathrm{~mW}$. Le faisceau est focalisé sur 1'échantillon. La lumière diffusée est collectée par une lentille au point focal de laquelle se trouve un trou calibré servant de filtre spatial, puis est rendue parallèle par une seconde lentille avant d'entrer dans l'interféromètre. Elle traverse alors un second filtre spatial avant d'être détectée par un photomultiplicateur. Une rampe électronique, en agissant sur 
des cales piézoélectriques, permet de faire défiler deux ordres d'interférence toutes les dix secondes. Après détection, le signal est analysé par un compteur de photons, puis stocké dans un analyseur multicannaux. Le balayage de celui-ci est synchronisé avec le balayage de l'interféromètre, de sorte que le résultat de chaque analyse s'ajoute de façon cohérente aux résultats accumulés précédemment. L'intervalle spectral libre de l'interféromètre est choisi de façon a ce que les raies Brillouin provenant des diamants de la cellule haute pression $\left(\Delta \sigma_{\mathrm{D}} \simeq 5,4 \mathrm{~cm}^{-1}\right)$ soient cachées par la raie Rayleigh.

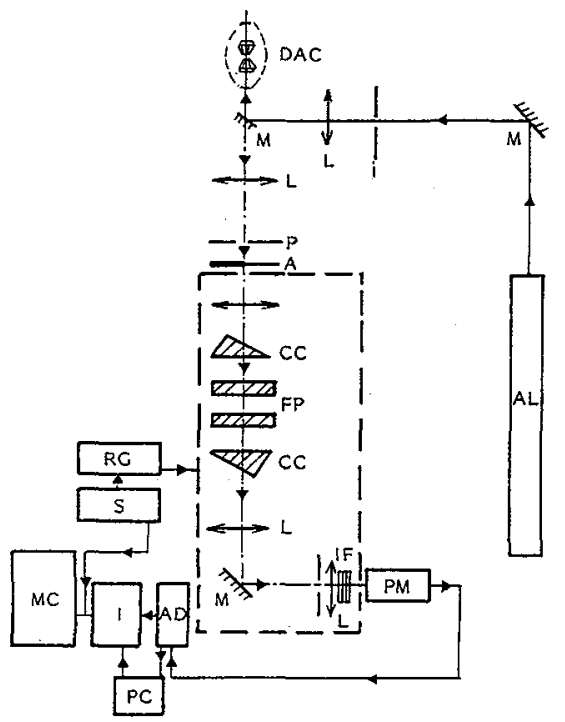

Fig. 2 - Dispositif de spectrométrie Brillouin en cellule à enclumes de diamant. AL, laser $\mathrm{Ar}^{+} ; \mathrm{M}$, miroir; $\mathrm{DAC}$, cellule à enclumes de diamant; $\mathrm{L}$, lentille; $\mathrm{P}$, trou calibrè; A, atténuateur; CC, coin de cube; FP, Fabry-Pérot; IF, filtre interférentiel; PM, Photomultiplicateur; $P C$, compteur de photons; $A D$, amplificateur discriminateur; $I$, interface; $R G$, rampe électronique; S, stabilisation; MC, calculateur.

\section{3 - PROPRIETES ELASTIQUES DE $\underline{\mathrm{SrTiO}}_{3}$ SOUS HAUTE PRESSION}

$\mathrm{SrTiO}_{3}$ est considéré comme un modèle pour l'étude des transitions de phase ferroélastiques. Une telle instabilité intervient dans ce corps à la pression atmosphérique et à la température critique $T_{c} \simeq 105 \mathrm{~K} / 4 /$, faisant ainsi passer le cristal d'une phase haute température de symétrie cubique (groupe d'espace $0_{h}^{1}$ ) vers une phase basse température de symétrie quadratique (groupe d'espace $D_{4 h}^{18}$ ). La pression ayant pour effet d'augmenter plus rapidement les forces à courte portêe que les interactions coulombiennes, la température critique augmente avec la pression et l'observation de la transition de phase est possible à la température ambiante (figure 3 ).

La mesure de $\Delta \sigma$ permet de déterminer la combinaison linéaire de constantes élastiques attachée aux modes observés. Pour cela on doit connâ̂tre l'équation d'état du cristal ainsi que I'indice du milieu. La densité de $\mathrm{SrTiO}_{3}$ a été mesurée par diffraction-X sur poudre en cellule à enclumes de diamant /5/. L'indice de réfraction a été calculé en utilisant la loi (P en GPa) /6/:

$$
n=2,4642-1,2 \cdot 10^{-3} P
$$


Dans le cas du mode transversal et dans la phase cubique, la combinaison ainsi déduite de nos mesures de $\Delta \sigma$ est $\left(C_{11}+C_{44}-C_{12}\right) / 3$.

Les résultats de la figure 4 correspondent à une direction de propagation parallèle à la direction [111] de la maille cubique. La transition cubique-quadratique a pu être observée à environ $6 \mathrm{GPa}$, ce qui est en accord avec I'extrapolation vers les hautes pressions du diagramme des phases /7/. Un mode transverse a pu être suivi jusqu'à $25 \mathrm{GPa}$; un mode longitudinal ou quasi longitudinal de la phase haute pression a pu être observé jusqu'à 36 GPa.
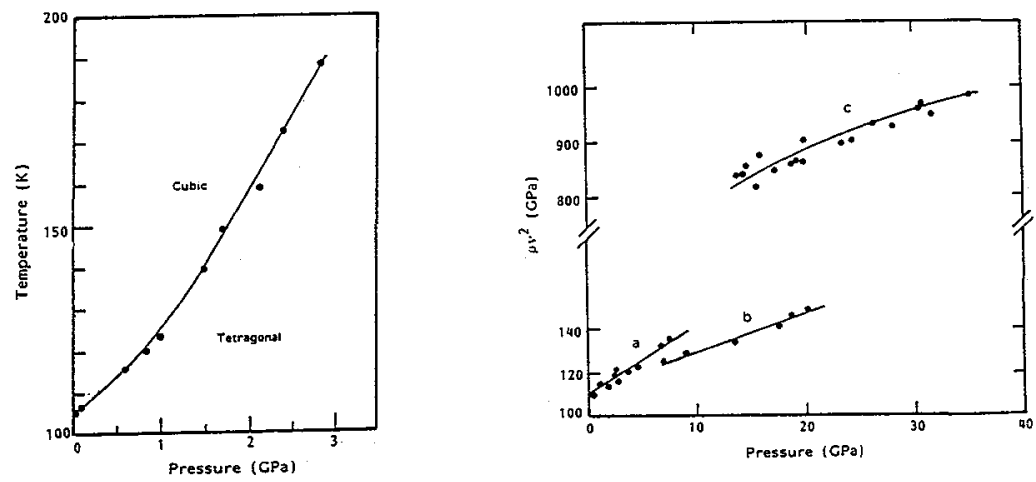

Fig. 3 - Diagramme des phases de $\mathrm{SrTiO}_{3}$ (d'après la référence /7/)

Fig. 4 - Variations des combinaisons linéaires de constantes élastiques en fonction de la pression. La direction de propagation est parallèle à la direction [111] de la phase cubique. Les courbes $a$ et $b$ montrent respectivement la dépendance en pression d'un mode transverse (phase cubique) et quasi-transverse (phase quadratique). La courbe c décrit la dépendance en pression d'un mode quasi-longitudinal.

4 - CONCLUSION

La diffusion Brillouin se présente comme un outil idéal pour déterminer avec précision les vitesses du son dans les matériaux transparents. Elle est particulièrement adaptée aux petits échantillons et fournit des informations sur les modes acoustiques dont la fréquence est inaccessible aux techniques ultrasonores. Toutefois, l'obtention de pics Brillouin bien définis peut nécessiter l'accumulation d'un nombre élevé de spectres (jusqu'à 200 dans le cas de nos expériences) et requiert 1'utilisation d'un Fabry-Pérot multipassage.

\section{REFERENCES}

/1/ Jayaraman, A., Rev. Sci. Inst. 57 (1986) 1013

/2/ Mao, H.K., Bell, P.M., Shanar, J.W. and Steinberg, D.J., J. Appl.

Phys. 49 (1978) 3276

/3/ Sandercock, J.R., RCA Rev., 36 (1975) 89

/4/ Unoki, H. and Sakudo, T., J. Phys. Soc. Jpn., 2 (1967) 546

/5/ Fischer, M., Bonello, B., Polian, A. and Léger, J.M., in American Geophysics Union Monograph on Perovskites (1989) 125

16/ Samara, G.A., Phys. Rev., 151 (1966) 378

/7/ Okai, B. and Yoshimoto, J., J. Phys. Soc. Jpn., 39 (1975) 163 